\title{
Biomimetic insect infochemical communication system
}

\author{
M. Cole, J.W. Gardner, Z. Rácz, S. Pathak \\ University of Warwick \\ Coventry, United Kingdom \\ M.Cole@warwick.ac.uk
}

\author{
T.C. Pearce, J. Challiss, D. Markovic \\ University of Leicester \\ Leicester, United Kingdom
}

\author{
A. Guerrero, L. Muñoz, G. Carot \\ Department of Biological Organic Chemistry \\ Consejo Superior de Investigaciones Científicas \\ Barcelona, Spain \\ B.S. Hansson, S. Olsson, L. Kübler \\ Max Planck Institute for Chemical Ecology \\ Jena, Germany
}

\author{
J. G. E. Gardeniers, N. Dimov, W. Bula \\ MESA+ Institute for Nanotechnology \\ University of Twente \\ Enschede, The Netherlands
}

\begin{abstract}
By exploiting recent advances in both our understanding of pheromone biosynthesis pathways and the detection of molecules in the insect nervous system, we are developing a novel communication system based on functional equivalents of the cellular, sub-cellular and molecular biological machinery. This will form the basis of a new branch of information technology employing infochemical ligands for communication over space and time. In order to achieve this objective, both chemical signal generation and reception are replicated by taking a systematic approach that implements reaction steps as biosynthetic modules, which are then hierarchically integrated as a technological solution towards realising 'infochemical communication'.
\end{abstract}

\section{INTRODUCTION}

The world of insects is awash with chemical information in the form of volatile semiochemicals as eusocial insects rely heavily on chemical communication to organize colonies, find food, locate conspecific mates and divide labour [1]. Pheromone mediated chemical communication in insects provides the key form of information exchange between individuals and the chemical cues often have associated behavioural changes via the neuroendocrine function. These semiochemicals are complex and diverse as most species rely upon a number of different compounds to convey specific information [2]. This complex form of information exchange in invertebrates, mediated by chemicals, represents an unexplored form of communication and labelling technology that is yet to be exploited.

In this paper we report on our preliminary results on our study of the biosynthesis of pheromones and their detection as a basis for developing new synthetic biological subsystems. A generic configuration of the biosynthetic modules forming an infochemical communication system is shown in Figure 1. The combined biosynthesis (exocrine) subsystem forms a 'chemoemitter'- a microsystem capable of generating a precise mix of predefined volatile compounds (e.g. sex pheromones) in precise programmable ratios of vapour concentration.

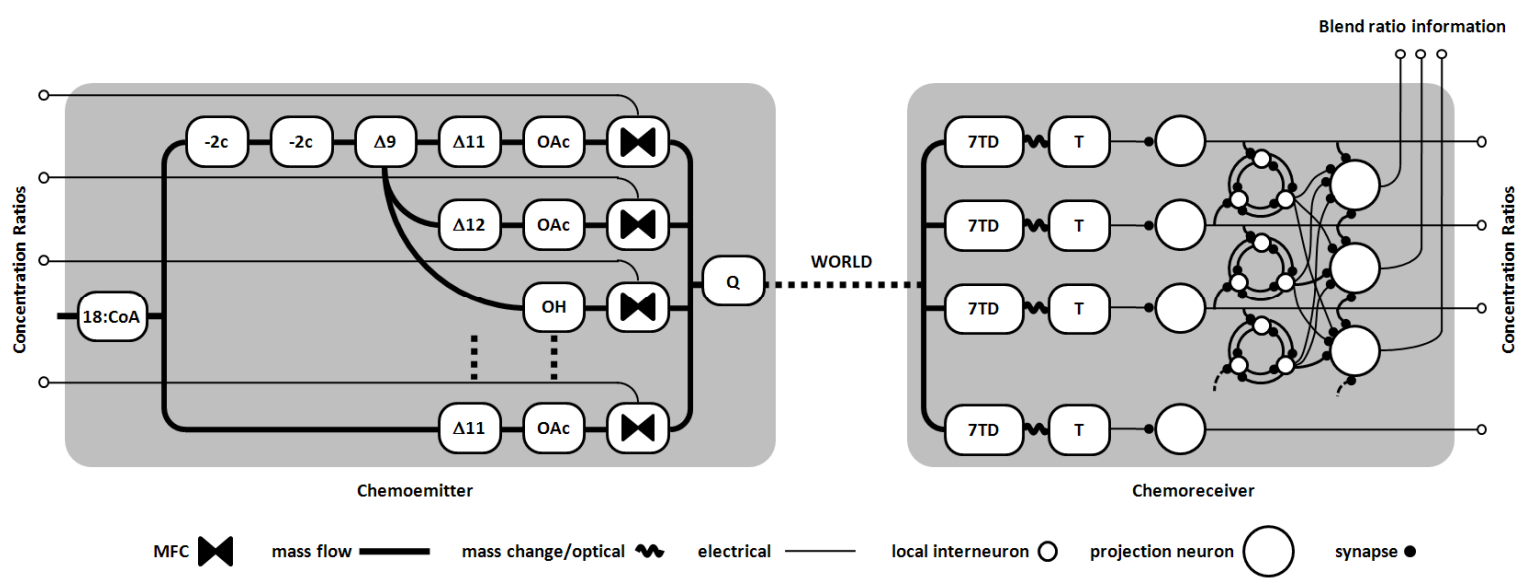

Fig. 1. Schematic diagram of a possible configuration of biosynthetic modules forming an infochemical communication system. The chemoemitter exploits different types of biosynthetic modules for the production of infochemicals based upon known enzymatic activity within the exocrine system of a moth. The piezoelectric actuator $(\mathrm{Q})$ releases the infochemical blend from a surface or emits a fine aerosol. The chemoreceiver exploits signalling from the olfactory receptors (e.g. 7 transmembrane), which are transduced (T) using binding specific changes. Infochemical binding produces highly specific signals to the known moth infochemical components which are processed in a ratiometric neuronal model based on the antennal lobe of the same animal. 
The combined detection system comprises olfactory receptors, for example a 7-transmembrane domain (7TD) transduction, and subsequent neuronal processing architecture with local interneurons to form the ratiometric chemoreceiver.

The objective of the work presented is to engineer novel functional equivalents of the molecular, subcellular and cellular machinery comprising the pathway between pheromone production and detection in insects. In this paper we present developments realized so far towards the implementation of a prototype consisting of a biosynthetic chemoemitter and chemoreceiver.

\section{BIOSYNTHETIC MODULE DEVELOPMENT}

The moth Spodoptera littoralis has been chosen as the biological model for studying pheromone communication because (1) there are at least two distinct pheromone components known in this animal that are well defined Z9,E11-14:OAc and Z9, E12-14:OAc as well as a behavioural antagonist Z9-14:OH, (2) the detection pathways including the receptors have been extremely well characterized in this insect. In fact, it is known that the male moth is attracted to a specific ratio of a mixture of five different pheromones.

Both chemical signal generation and reception has been considered in this work by taking a systems approach in which each process in the pheromone generation and detection pathway is deployed as a discrete biosynthetic module.

\section{A. Chemoemitter}

Most of the pheromone compounds in Spodoptera littoralis are simple structures consisting of a hydrocarbon chain and oxygenated functional group, typically an alcohol $(\mathrm{OH})$, aldehyde (Ald), hydrocarbon ( $\mathrm{Hc})$, acetate ester (OAc) or epoxide (Epox), produced in four clearly defined steps that are mediated by enzymes: fatty acid synthesis, desaturation, hydrocarbon chain shortening, and reduction/functional group modification.

Each biological process identified as part of the biological pathway of pheromone production in Spodoptera littoralis [3] is characterized and implemented in a modular microchip system. The microreactor modules produce intermediate chemical species and combining the modules in a hierarchical structure enables the production of an almost limitless diversity of pheromone compounds. Each microreactor module is designed to facilitate the immobilization of the key enzymes involved in pheromone synthesis whilst preserving catalytic activity and provides accurate control of the concentration and residence time of the reactants and endproducts. The synthesized pheromone blends are released into the environment by a temperature controlled micro-evaporator module (Figure 2).

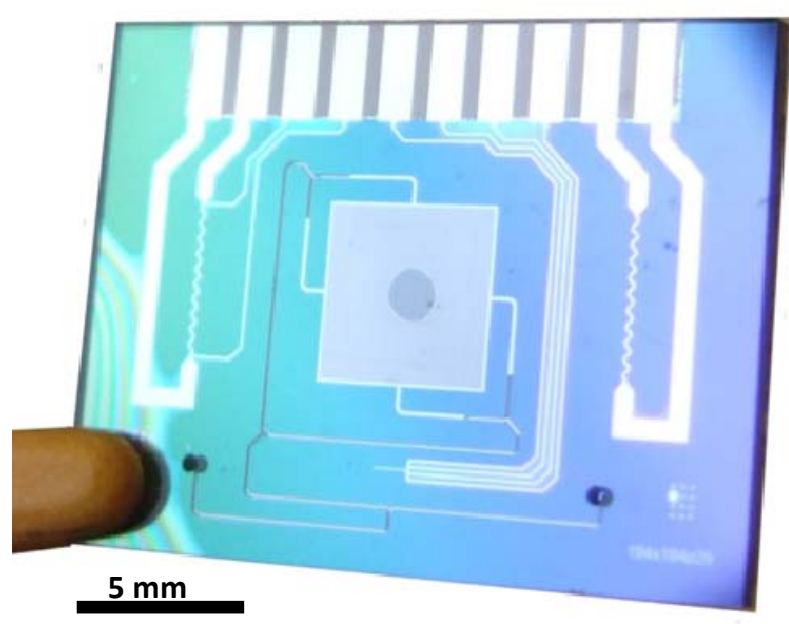

Fig. 2. Silicon/glass micromachined artificial gland for pheromone release.

In the micromachined evaporator - referred to here as an "artificial gland" - microfluidic channels transport the pheromone and the diluting agents to the release area that is made of a perforated silicon membrane covered with black silicon. Black silicon was selected as the surface material because evaporation measurements made on different microstructured surfaces (i.e. polished silicon, porous silicon, black silicon and carbon nanofibres) showed that black silicon provides the highest rate of evaporation of pheromone components at $c a .2 .5 \mathrm{ng} / \mathrm{s}$.

A crucial aspect of the artificial gland design is the evaporation rate of the major pheromone components. The loss of mass from a $1 \mathrm{~cm}^{2}$ black silicon surface due to evaporation with time is shown in Figure 3. The measurements were taken in a wind tunnel with an air velocity of $20 \mathrm{~cm} / \mathrm{s}$ and loss of material quantified by gas chromatography.

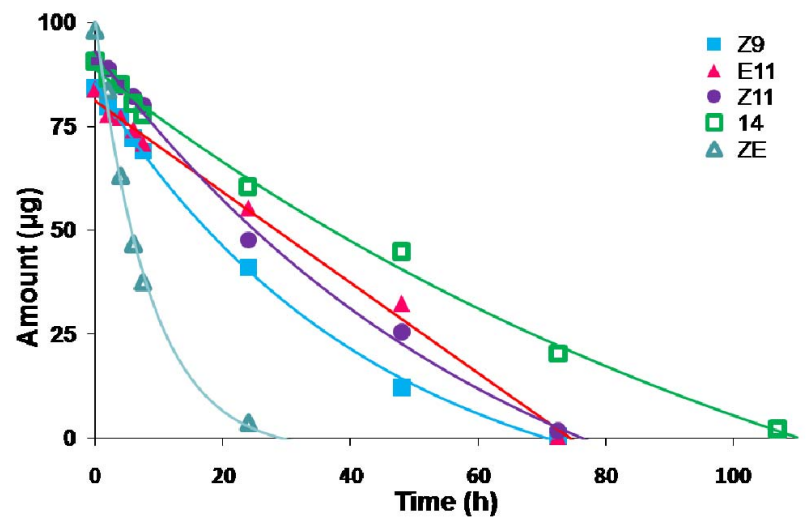

Figure 3. Plot showing the loss of mass of the major pheromone compounds vs. time due to evaporation in a wind tunnel.

\section{B. Chemoreceiver}

Insects need to be able to detect specific ratios of pheromone cues within their environment as a cue for for- 
aging, mate location, and prey avoidance. This process invokes a whole cascade of events before and after receptor activation. Odour molecules striking the sensilla absorb onto its waxy surface and subsequently diffuse through the cuticle to interact with a number of special purpose proteins [4].

The key specificity in the response to pheromones is considered to be due to the specificity of ligand binding olfactory receptors. Molecular recognition of a diverse set of ligands is achieved through a large family of proteins encoded by olfactory receptor (OR) genes. Four putative pheromone receptor candidates for $S$. littoralis have been identified [5].

The initial work to develop a new biosynthetic module for olfactory receptors and transmembrane domain signalling is focused on the use of heterologous expression systems, such as human embryonic kidney (HEK) cells, in which these ORs can be efficiently expressed and then coupled to artificial systems for ligand detection. To that effect, custom surface acoustic wave (SAW) devices and microfluidic components, shown in Figure 4, have been designed and a protocol for the deposition and characterization of HEK-293 cells on SAW devices (Figure 5) developed.

Ligand detection is achieved by monitoring the ligand binding-related changes in the interaction between the cell and the acoustic waves generated by the piezoelectric device. In order to probe all regions of interest in the HEK cell membrane and the cell itself, several device configurations of different operating frequencies, thus different sensing distances, have been designed. Each SAW device is a dual delay-line or dual resonator sensor system to allow differential measurements in which only one device of the pair is functionalized (e.g. with OR-expressed HEK-293 cells) and the other serves a reference. In this way, common mode signal interferences such as temperature or humidity drift can be obviated.

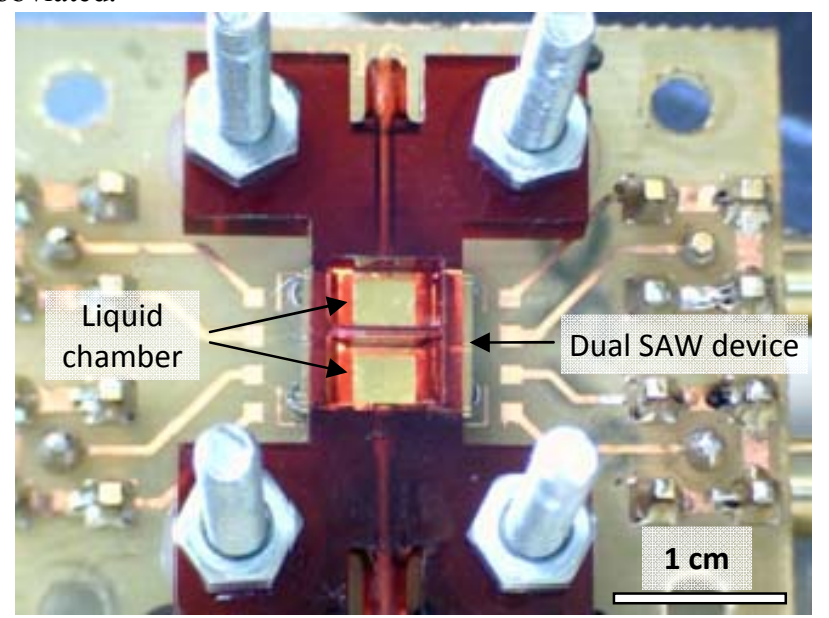

Fig. 4. Dual surface acoustic wave delay line device and plastic liquid chamber mounted on a printed circuit board.

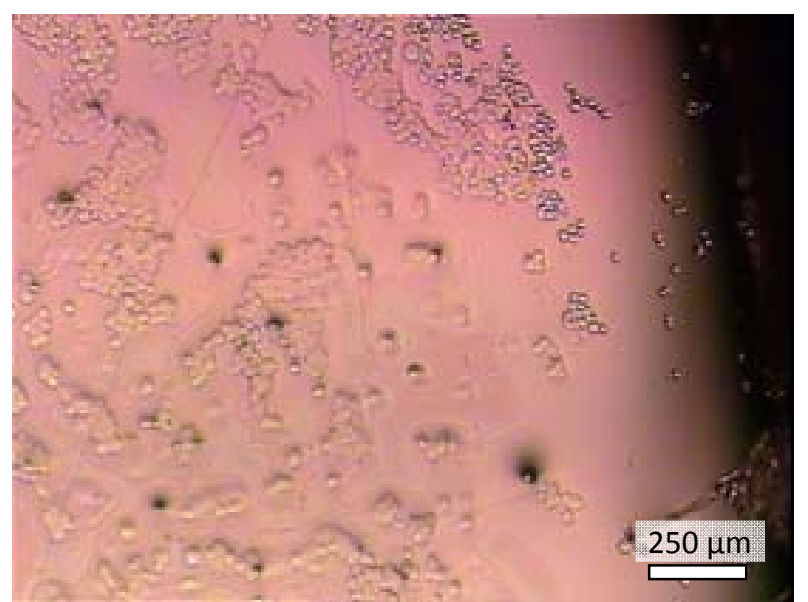

Fig. 5. Optical microscope image of human embryonic kidney cells grown on top of a gold coated lithium tantalate SAW device.

Surface acoustic wave devices and their coatings do not have highly specific chemical responses. Therefore, in order to process SAW receptor signals and relate them to ratio decoding and blend recognition, a biophysically constrained neuronal model mimicking the ratiometric processing in the antennal lobe of Lepidoptera has been developed and implemented (Figure 6). The olfactory receptor neurons (ORNs) depicted by green and yellow triangles, respectively, make excitatory synapses (green and yellow circles) with the local interneurons (LNs). Local interneurons each have a probability to form inhibitory interconnections to other LNs, as illustrated by the dark blue circles from the output of the local neurons. Excitatory inputs from the ORNs and inhibitory inputs from LNs converge to an adder that superimposes the spike trains from multiple sources to drive the target. The pheromone model also contains two glomeruli, however, unlike the local interneurons that receive input from every ORN, each glomerulus only receives input from a particular ORN. Principal neurons (PNs) can also make excitatory connections toward PNs within a single glomerulus, but connections toward another glomerulus are not allowed.

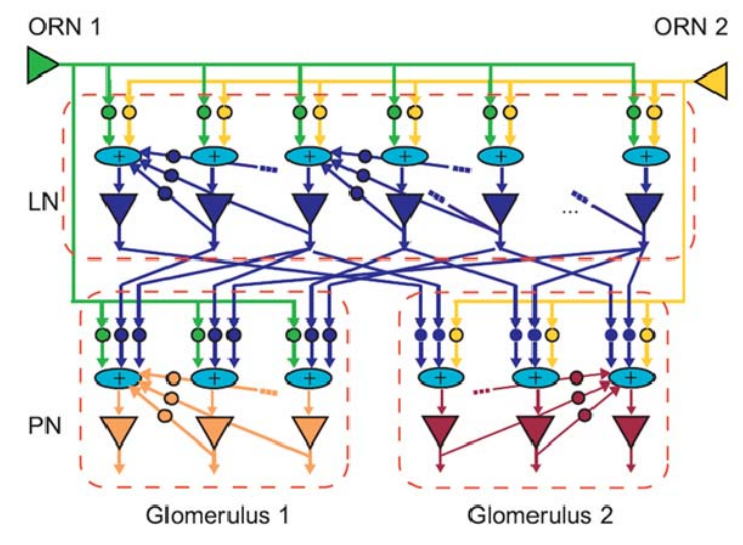

Fig. 6. Schematic representation of the antennal lobe ratio decoding model. The triangles represent integrate-and-fire cells and the circles represent dynamical synapses.

This research is funded by the European Community's $6^{\text {th }}$ Framework

Programme - iCHEM project. 
This arrangement of local interneurons allows more than one glomeruli to respond to one ORN (sensor) and thus permits the encoding of ratios of different ligands. Classification takes place using the outputs of the PNs. Combining the olfactory signalling module with the neuronal processing model deployed in silicon will complete the development and implementation of the novel chemoreceiver unit.

\section{SYSTEM INTEGRATION}

In parallel with designing, fabricating and characterizing the chemoemitter and the chemoreceiver subsystems, a proofof-concept prototype integrating all components has been developed, shown in Figure 7. The functionality of this combined infochemical communication system has been demonstrated using piezoelectric atomizers with presynthesized compounds as emitters, an odour chamber as controlled environment for chemical transfer and a quartz crystal microbalance-based functionalized sensor array as receiver. Interface electronics and LabVIEW (National Instruments Corp., Austin, USA) software facilitate direct computer control and data acquisition via USB ports.

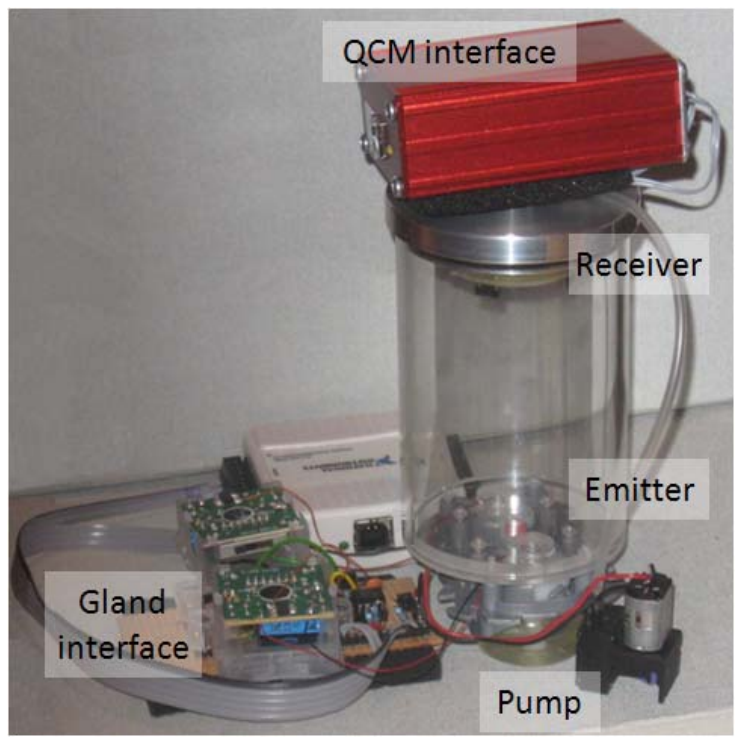

Fig. 7. Prototype system consisting of a piezoelectric atomizer serving as an artificial gland, a glass odour chamber and a sensor array of four quartz crystal microbalance-based micro-chemoreceivers. Also shown are the interface circuits that enable direct computer control and data acqusition via USB ports.

In parallel with the development of the novel SAW-based infochemical communication system, integration of the SAW sensor and the associated interface circuitry into a single monolithic microelectronic system is underway. The schematic block diagram of the proposed integrated system is shown in Figure 8.

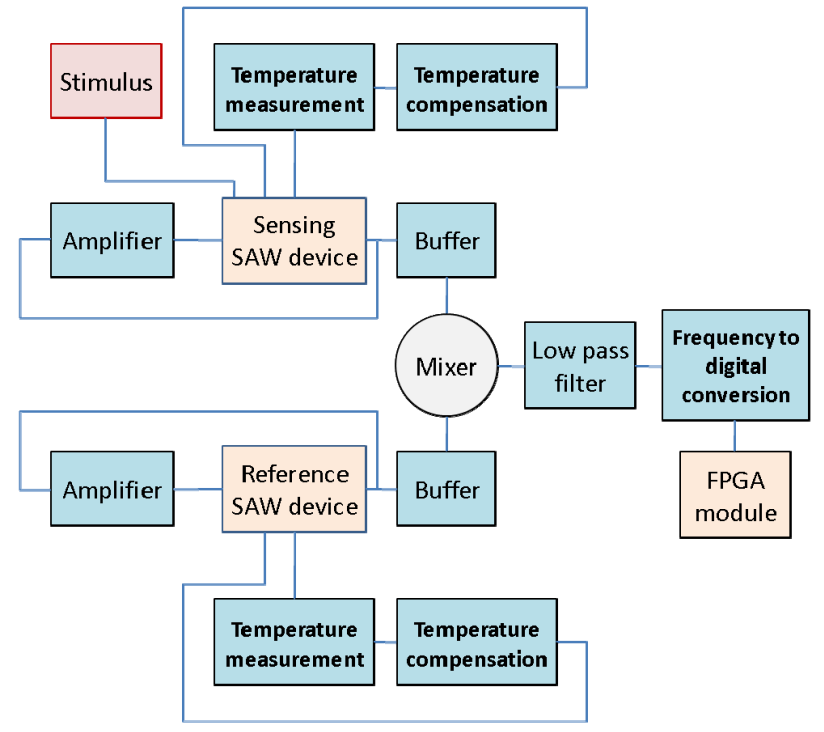

Fig. 8. Schematic block diagram of the integrated surface acoustic wave sensing system. The odour stimulus is detected as the differential frequency response of a temperature compensated SAW sensor pair arranged in oscillator configuration. The generated signal provides the input of the neuromorphic olfactory model implemented in the FPGA module.

High precision ligand detection is achieved by employing two temperature-compensated SAW sensors - one serves as the sensing device with transfected HEK cells while the other is used as reference HEK cells. The differential output of the sensing and the reference oscillator is connected to the input of a mixer, thus, the much lower frequency difference can be extracted and transmitted along normal wires for conversion to digital signals and further processing.

\section{CONCLUSIONS}

Infochemical communication is an emerging field and has potential application in diverse areas from pest management to group coordination of swarms of mobile robots. Here we have presented preliminary results following the synthetic modelling of the biology of the moth Spodoptera littoralis and fabrication of infochemical microfluidic systems towards a "moth-on-a-chip".

\section{REFERENCES}

[1] K. Von Frisch, The Dance Language and Orientation of Bees, Oxford University Press, London, UK, 1967.

[2] C. E. Linn Jr. and W. L. Roelofs, "Pheromone communication in moths and its role in the speciation process," in Speciation and the Recognition Concept - Theory and Application, D. M. Lambert and H. G. Spencer, Eds. The John Hopkins University Press, Baltimore, USA, 1995.

[3] L. Muñoz, G. Rosell, C. Quero and A. Guerrero, "Biosynthetic pathways of the pheromone of the Egyptian armyworm Spodoptera littoralis," Physiol. Entomol., vol. 33, pp. 275-290, 2008.

[4] J. G. Hildebrand and G. M. Shepherd, "Mechanisms of olfactory discrimination," Annu. Rev. Neurosci., vol. 20, pp. 595-631, 1997.

[5] E. Jacquin-Joly, private communication, 2008. 